\title{
Quality of Communication between Cancer Patients and Doctors
}

\author{
Zatul Himmah Mokhtar¹, Li Ping Wong², Mei Chan Chong,,, Abdulaziz Mansoor Al Raimi', Latifa Yahya Al Ajeel ${ }^{5}$ \\ and Samira Mohajer ${ }^{6}$ \\ 1Department of Nursing Science, Faculty of Medicine, Universiti Malaya, Malaysia \\ 2Professor, Department of Social and Preventive Medicine, Faculty of Medicine, Universiti Malaya, Malaysia \\ ${ }^{3}$ Associate Professor, Department of Nursing Science, Faculty of Medicine, Universiti Malaya, Malaysia \\ ${ }^{4}$ Department of Nursing Science, Faculty of Medicine, Universiti Malaya, Malaysia. Seiyun Community College, Yemen \\ ${ }^{5}$ Department of Health Sciences, Seiyun Community College, Yemen \\ ${ }^{6}$ Department of Nursing Science, Faculty of Medicine, Universiti Malaya, Malaysia. b Nursing and Midwifery Care Research Center, Mashhad University of Medical \\ Sciences, Mashhad, Iran
}

* Corresponding author: Mei Chan Chong, Department of Nursing Science, Faculty of Medicine, Universiti Malaya, Malaysia. Email: mcchong@um.edu.my

Received 2021 September 01; Revised 2021 December 04; Accepted 2021 December 14.

\begin{abstract}
Background: Communication between cancer patients and healthcare providers plays a vital role in providing a better quality of life for cancer patients. However, it remains a debatable issue in society.

Objectives: This study aimed to determine the quality of communication (QOC) between cancer patients and doctors and its associated factors.

Methods: This cross-sectional study was performed on a total of 600 cancer patients in the oncology clinic admitted to the oncology ward. A 19-item QOC questionnaire was administered on patients, along with measuring how patients rate the quality of this communication about endof-life care to improve communication between doctors and their patients.

Results: The cancer patients rated their doctors highly at "Including your loved ones in decisions about your illness and treatment", "Caring about you as a person", and "Answering all your questions". The areas that cancer patients rated relatively low included "Most doctors do not discuss how long the cancer patients might live" and "What dying might be like". The mean score for the overall doctor's QOC was obtained at $8.23 \pm 0.74$. This indicated that the level of doctor communication was close to very good communication. The following factors contributed remarkably on the scores of QOC with doctors: being 71 years old and above, holding secondary school qualifications, being diploma/degree holder, working for the government, private sector, factory, or estate, and being self-employed, being diagnosed with cancer for more than 1 year, lacking monthly income, and not staying with family.

Conclusion The results of the study showed that socio-demographic factors of cancer patients, including age, education level, employment status, working sector, illness duration, and income, could affect communication, most of the time, negatively. This issue should be taken seriously for the improvement of the care of cancer patients.
\end{abstract}

Keywords: Cancer patient, Doctors communication, Effective communication, End-of-life care, Healthcare providers, Quality of communication

\section{Background}

Currently, the second greatest cause of mortality worldwide is cancer after ischemic heart disease. Cancer fatalities are currently more prevalent than cardiovascular disease mortality in high-income nations (1). 7In Malaysia, cancer incidence is expected to double by 2040 . Understanding cancer awareness is important to adopt preventative measures and reduce the cancer burden. Although reasons for cancer development are not clear, the results of prior studies have shown that cancer patients have a lack of knowledge and awareness about the sign and symptoms, treatment, and prognosis. Cancer patients may receive lower quality of end-of-life (EOL) care since cancer patients-doctors communication may be less likely to occur and more difficult to conduct for patients with a less certain prognosis (2).

Communication is one of the important components in providing quality care for cancer patients. To ensure exceptional cancer care, the communication between patient and clinician should emphasize the diagnosis, prognosis, possible treatments, and patient's treatment choice (3). Effective communication can help to lighten patients' anxiety, depression, and other emotional problems that might act as pain relief of other symptoms and improvement of cancer patients' quality of life. Apart from that, effective communication can empower cancer patients and enhance their participation in selfcare and treatment management (4). In order to improve communication about the quality of EOL care for cancer patients, it would be useful to identify the specific components of this communication to identify targets for future interventions.

Despite the advances in cancer care, the approaches towards efficient communication with cancer patients are somehow absent. Walczak et al. (5) reported that topics related to prognosis and EOL care might be challenging for doctors and cancer patients to talk about. Faller et al. (6) supported this by reporting that it was most difficult to discuss the bad news, palliative care, and spiritual issues and talk to patients or families from multiple socio-demographic 
backgrounds.

Socio-demographic backgrounds among patients affect the popularity of symptoms; the edge for seeking care; the willingness and skill to speak and explain about the symptoms; the understanding of ordinary information about the diagnosis, prognosis, and treatment options; the trust in several professionals; and therefore, adherence to prescribed treatments (7). Surbone (8) emphasized that the socio-demographic factors significantly contribute to the communication problem between the oncologists and patients. Smooth communication can be achieved by consistent engagement with the patients during their care as this helps in understanding their personal preferences. Brown et al. (9) reminded the healthcare professionals in cancer care that socio-demographic elements were able to impact the communication. Battling cancer is particularly stressful and emotional for the patients and affects their quality of life (10). Therefore, effective communication is the central element for doctors to supply quality care and ensure that the care provided is according to the patients' values and priorities. Once the health care team of cancer patients decided that the disease is uncontrollable, the medical testing will be terminated; nonetheless, the care will be provided continuously with a stress on improving their EOL care and making them comfortable for the subsequent years, months, or weeks (11).

However, the importance of communication between patients and healthcare providers is becoming increasingly widespread outside of the inpatient situation. When the patient is no longer under the care of a health care professional, the care team should remain in touch with patients to ensure safe outcomes and meanwhile strengthen forming meaningful relationships (10).

Predominantly, in oncology, communication skills are crucial in gaining information regarding the patients' reactions to their diagnosis (12). In a medical situation, communication focuses not only on sharing information about problems, causes, and treatments but also on acknowledging the emotional needs of patients (13). Brueck and Salib (14) have stated that once patients realize that the healthcare providers are truly concerned about them, they will be more satisfied with their medical consultation. Better understanding should be achieved between the two parties to guarantee the quality of the given care. The findings of a study reported that effective communication positively affected the speed of patient recovery, pain control, adherence to treatment regimens, psychological functioning, and quality of life (15). This study aimed to determine the quality of communication $(\mathrm{QOC})$ between cancer patients and doctors and its associated factors.

\section{Objectives}

This study aimed to determine the quality of communication (QOC) between cancer patients and doctors and its associated factors.

\section{Methods}

This descriptive cross-sectional study was carried out at a teaching hospital in Malaysia. The study population included cancer patients enrolled in the oncology clinic and admitted to the oncology ward. The inclusion criteria were cancer patients being diagnosed by any cancer variations, receiving cancer treatment, and being 18 years old and above. On the other hand, patients being unable to communicate verbally or having incapacitated mentally were excluded from the study.

The participants $(n=600)$ were selected using the convenience sampling method. The sample size was determined based on a 5\% standard error and 95\% confidence interval, as used by the Raosoft sample size calculation, and the estimated proportion formula. Consequently, the sample size of the present study was based on a formula. The general rule relative to acceptable margins of error in survey research is 5$10 \%$. The estimated population for this study was 40,000 cases, which was based on the yearly registration of cancer patients. The researcher used a $50 \%$ response rate as it required a larger sample size to generalize the results of the study. This was considered to exclude those patients who might drop out from the study or are unable to complete the questionnaires during the study period.

\subsection{Data Collection}

Part one was designed to collect the demographic data of cancer patients. This information included gender, ethnicity, age, highest education level, employment status, working sector, illness duration, monthly income, and living place. Part two measured the QOC with the doctors. The questionnaire was adapted from the 'Psychometric Characteristics of a Quality of Communication' questionnaire from the Journal of Palliative Care and Medicine (16).

This 19-item tool evaluates satisfaction on the QOC between cancer patients and doctors, with specification on EOL ( $\mathrm{n}=18$ items) and measures the overall QOC ( $\mathrm{n}=1$ item). It was validated in a study conducted by Engelberg et al. (2006) (16). Each participant was also asked to identify the doctors who were primarily responsible for taking care of them.

The instrument was adapted in relevant to Malaysian cultural settings. Patients have to rate the doctors' communication skills on a scale of 0 to 10 , and the maximum score is obtained at 180 . The scores of $0-60,61-120$, and 121-180 indicate poor, good, and excellent communication, respectively. These items are rated on a Likert scale, and continuous (e.g., 1-10) rating scales are quantitative. These scales assume equal intervals between points. Furthermore, this method of reporting is also quite useful for evaluating 
a respondent's opinion on satisfaction features that can be gradually represented (17).

The bilingual questionnaire was used. The translation of the instrument to the local language is essential to conduct cross-cultural research with nonEnglish speaking populations. The original English questionnaire was translated to Bahasa Malaysia by using forward and backward translation procedures. Based on the guideline provided by the World Health Organization (18), the procedures of forward and backward translation include forward translation, expert panel back-translation, pre-testing, cognitive interviewing, and final version.

A panel consisting of two local oncologists and three nurse managers from the oncology clinic, oncology ward, and palliative ward were chosen to evaluate the content validity. After the confirmation of the validity of the research tools, they received no other changes. The questionnaire underwent a pilot study that involved 150 cancer patients from 3 ethnic groups, namely Malay, Chinese, and Indian. Cronbach's alpha coefficient method was employed to estimate the internal consistency reliability of the QOC Questionnaire. Based on the result of the pilot study, Cronbach's alpha coefficient was obtained at the range of 0.719-0.860; therefore, no changes were made in the original questionnaires since all participants understood each statement in the items of the questionnaire.

\subsection{Ethical considerations}

Ethical approval was obtained from the Research Ethics Committee (Reference number: MECID.NO: 20147-361). The research objectives and procedures were explained to all individuals in written form, and they were assured of anonymity and confidentiality in this study. In this respect, each participant was coded with numbers during the study.

\subsection{Data analysis}

Data were analyzed in Statistical Package for Social Science (SPSS) statistic version 22.0. Inferential statistical tests, such as t-test and one-way ANOVA, were used to determine the differences between demographic variables. On the other hand, the multivariate linear regression model was applied in deciding the predictors of QOC.

\section{Results}

\subsection{Demographic characteristics}

The demographic characteristics of respondents are presented in Table 1. More than half of the respondents were female, and Chinese was the dominant ethnicity, followed by Malay, and Indian. Regarding the age variable, it was discovered that twothird of the cancer patients aged 41-70 years old. In terms of the highest level of education, about half of the cancer patients held a secondary school degree. It

\begin{tabular}{lc}
\hline Table 1. Socio-demographic characteristics of cancer patients (n=600) \\
\hline Variable & $\mathbf{n}(\mathbf{6})$ \\
\hline Gender & $256(42.7)$ \\
Male & $344(57.3)$ \\
Female & \\
Ethnicity & $196(32.7)$ \\
Malay & $284(47.3)$ \\
Chinese & $120(20.0)$ \\
Indian & \\
Age (years) & $106(17.7)$ \\
21-40 & $399(66.5)$ \\
41-70 & $95(15.8)$ \\
>71 and above & \\
Education & $124(20.7)$ \\
Primary School & $310(51.6)$ \\
Secondary School/Certificate & $166(27.7)$ \\
Diploma /Degree & \\
Employment status & $327(54.5)$ \\
Employed & $273(45.5)$ \\
Not Employed & $136(22.7)$ \\
Working sector & $224(37.3)$ \\
Government & $240(40.0)$ \\
Private/Self-employed/Factory/Estate & \\
Retired & $263(43.8)$ \\
Illness duration & $254(42.3)$ \\
Newly diagnosed & $83(13.9)$ \\
First year & $154(25.7)$ \\
>1 year & $179(29.8)$ \\
Monthly income & $267(44.5)$ \\
125-750 \$ & \\
No Salary & \\
Living place & \\
Yes & \\
No & \\
\hline
\end{tabular}


Table 2. Descriptive Analysis for doctor communication variable

\begin{tabular}{|c|c|}
\hline Item & $\mathrm{M} \pm \mathrm{SD}$ \\
\hline Including your loved ones in decisions about your illness and treatment. & $8.15 \pm 0.80$ \\
\hline Caring about you as a person. & $8.12 \pm 0.63$ \\
\hline Answering all your questions about your illness and treatment. & $8.09 \pm 0.74$ \\
\hline Listening to what you have to say. & $8.08 \pm 0.70$ \\
\hline Giving you his/her full attention. & $8.00 \pm 0.67$ \\
\hline Looking you in the eyes. & $7.95 \pm 0.76$ \\
\hline Asking about the things in life that are important to you. & $7.95 \pm 0.66$ \\
\hline Respecting the things in your life that are important to you. & $7.86 \pm 0.79$ \\
\hline Using words that you can understand. & $7.69 \pm 0.89$ \\
\hline Involving you in the decisions about the treatments that you want if you get too sick to speak yourself. & $7.69 \pm 0.85$ \\
\hline How comfortable do you feel your doctor is talking about dying? & $7.69 \pm 0.76$ \\
\hline Talking with your loved ones about what your dying might be like. & $7.61 \pm 1.00$ \\
\hline Talking with you about your feelings concerning the possibility that you might get sicker. & $7.57 \pm 0.84$ \\
\hline Asking about your spiritual or religious beliefs. & $7.44 \pm 0.75$ \\
\hline Respecting your spiritual or religious beliefs & $7.40 \pm 0.85$ \\
\hline Talking to you about the details concerning the possibility that you might get sicker & $7.36 \pm 0.87$ \\
\hline Talking to you about what dying might be like. & $7.28 \pm 0.99$ \\
\hline Talking to you about how long you might live. & $7.19 \pm 0.87$ \\
\hline Overall, how would you rate this doctor's communication with you? & $8.23 \pm 0.74$ \\
\hline
\end{tabular}

was also found that a total of 327 cancer patients were employed. Among the employed respondents, more than one-third were working in the private sector, factory, or estate, while the others were in the government sector.

\subsection{Quality of communication between cancer patients and doctors}

Table 2 summarizes the descriptive analysis of the doctor communication variable. A total of 18 items related to QOC were arranged from the highest to the lowest mean score. The highest mean scores were reported for the items "Including your loved ones in decisions related to your illness and treatment" and "Caring about you as a person". The lowest mean scores were determined for the items "Talking to you about what dying might be like", "Talking to you about how long you might live", and "Asking about your spirituality and respecting your spiritual or religious beliefs".

As shown in Table 3, the mean total score of QOC with doctors varied according to the patients' demographic characteristics. The mean total score of QOC with doctors was significantly higher in cancer patients aged above 71 years old. Furthermore, the highest mean total score was reported among diploma patients or degree holders.

Univariate analysis was carried out to evaluate the relationship between socio-demographic characteristics and the mean total score of QOC with doctors. Multivariate linear regression was then performed on the variables of demographic characteristics (independent variable) and tested the association between this variable and the total mean score of the QOC with doctors.

Based on the univariate analysis, significant associations $(\mathrm{P}<0.05)$ were keyed in the multivariate linear regression model. The result of the multiple linear regression showed that the following variables affected the scores of QOC with doctors: being 71 years old and above; holding secondary school qualification; being diploma/degree holder; working for the government, private sector, factor, and estate; and being self-employed (Table 3 ).

Table 3. Multivariate linear regression analysis of cancer patient's socio-demographic characteristics and quality of communication with doctors

\begin{tabular}{|c|c|c|c|c|}
\hline & \multicolumn{3}{|c|}{$\begin{array}{l}\text { Quality of communication } \\
\text { with doctors score }\end{array}$} & \multirow{2}{*}{$\begin{array}{c}\text { Multivariate linear regression of } \\
\text { quality of communication with doctors } \\
\beta(95 \% \mathrm{CI}) \\
\end{array}$} \\
\hline & N (\%) & Mean (SD) & p value & \\
\hline \multicolumn{5}{|l|}{ Gender } \\
\hline Male & $256(42.6)$ & $139.05 \pm 8.75$ & 0.868 & - \\
\hline Female & $344(57.2)$ & $139.71 \pm 8.80$ & & - \\
\hline \multicolumn{5}{|l|}{ Ethnicity } \\
\hline Malay & $196(32.6)$ & $140.64 \pm 8.70$ & & $0.56(-1.28-2.39)$ \\
\hline Chinese & $284(47.3)$ & $138.69 \pm 7.65$ & 0.007 & $0.56(-1.14-2.26)$ \\
\hline Indian & $120(20.0)$ & $137.64 \pm 11.02$ & & $\operatorname{Re}$ \\
\hline \multicolumn{5}{|l|}{ Age (years) } \\
\hline $21-40$ & $106(17.7)$ & $141.98 \pm 8.77$ & & $\operatorname{Re}$ \\
\hline $41-70$ & $399(66.5)$ & $137.21 \pm 7.25$ & $<0.001^{*}$ & $-0.01(-2.37-2.35)$ \\
\hline$\geq 71$ & $95(15.8)$ & $143.94 \pm 1.78$ & & $6.27(3.25-9.29)^{*}$ \\
\hline \multicolumn{5}{|l|}{ Highest education level } \\
\hline Primary School & $124(20.7)$ & $137.94 \pm 2.19$ & & $\operatorname{Re}$ \\
\hline Secondary School/Certificate & $310(51.7)$ & $138.47 \pm 7.05$ & $<0.001^{*}$ & $1.99(0.22-3.76)^{*}$ \\
\hline Diploma/ Degree & $166(27.6)$ & $141.20 \pm 8.48$ & & $4.14(1.81-6.48)^{*}$ \\
\hline
\end{tabular}




\begin{tabular}{|c|c|c|c|c|}
\hline \multicolumn{5}{|l|}{ Employment status } \\
\hline Employed & $327(54.5)$ & $137.40 \pm 7.32$ & $<0.001^{*}$ & - \\
\hline Not employed & $273(45.5)$ & $141.13 \pm 9.95$ & & - \\
\hline \multicolumn{5}{|l|}{ Working Sector } \\
\hline Government & $136(22.7)$ & $141.96 \pm 7.91$ & & $14.85(11.39-18.31)^{*}$ \\
\hline Private / Factory / Estate/ Self Employment & $224(37.3)$ & $136.21 \pm 7.50$ & $<0.001^{*}$ & $10.31(6.99-13.61)^{*}$ \\
\hline Retired & $240(40.0)$ & $140.23 \pm 9.65$ & & $\operatorname{Re}$ \\
\hline \multicolumn{5}{|l|}{ Illness duration } \\
\hline Newly diagnosed & 263(43.8) & $137.77 \pm 8.07$ & & $\operatorname{Re}$ \\
\hline First year & $254(42.3)$ & $138.74 \pm 8.17$ & $<0.001^{*}$ & $0.14(-1.23-1.51)$ \\
\hline$>1$ year & 83(13.8) & $139.12 \pm 8.81$ & & $7.07(5.10-9.03)^{*}$ \\
\hline \multicolumn{5}{|l|}{ Monthly income } \\
\hline No income & $154(25.7)$ & $141.33 \pm 10.01$ & & $12.25(8.99-15.50)^{*}$ \\
\hline $125-750 \$$ & $179(29.8)$ & $135.57 \pm 7.70$ & $<0.001^{*}$ & $-0.97(-2.86-0.91)$ \\
\hline$>750 \$$ & 267(13.8) & $138.88 \pm 6.52$ & $<0.001^{*}$ & $\operatorname{Re}$ \\
\hline \multicolumn{5}{|l|}{ Living place } \\
\hline Yes & $512(85.2)$ & $138.94 \pm 8.96$ & $0.003^{*}$ & $\operatorname{Re}$ \\
\hline No & $88(14.6)$ & $140.20 \pm 7.89$ & & $2.51(0.47-4.56)^{*}$ \\
\hline
\end{tabular}

$\mathrm{M}=$ Mean; SD = Standard Deviation,Re: references group

Note $* p<0.05$ was significant with the $p$-value of 0.009 at a $95 \%$ confidence interval.

\section{Discussion}

In the current study, the cancer patients rated their doctors highly at "Including your loved ones in decisions about your illness and treatment", "Caring about you as a person", and "Answering all your questions". The items that cancer patients rated relatively low were "Most doctors do not discuss how long the cancer patients might live" and "What dying might be like". Fournier et al. (19) reported that older patients become more sensitive (especially when they had a greater need for information), the information needed to be given repeatedly, and it was quite difficult for them to comprehend the information. Consequently, the patients could probably be distressed and feel burdened and older patients might not communicate properly because of hearing and cognitive problems (20). Therefore, healthcare providers who work with older patients require training. Although sympathy and kindness are important qualities for service providers, being able to understand the patients and possessing basic helping skills are also essential.

The findings of our study showed that sociodemographic factors, including age, level of education, employment status, working sector, illness duration, and the income of cancer patients could affect communication, most of the time, negatively. This result was consistent with those of a study conducted by Davis (21) reporting that cancer patients with better education who chose to work or with better income or insurance had the tendency to take part in discussions and ask more questions about their illnesses. Furthermore, according to the findings of a study performed by Street Jr and Mazor (22), healthcare providers communicated differently with cancer patients based on their working sectors and financial status. Therefore, this difference in communication might lead to disparities in QOC (23). It was also reported that low-income populations and patients without insurance coverage had lower communication satisfaction and access to care (22).
The results of our study also revealed that the duration of illness was significantly correlated with the cancer patient's QOC with doctors. According to the results of a study carried out by Hofmeister (24), cancer patients who were newly diagnosed often worried about their conditions and kept asking about their prognosis. Regarding this, it is important to practice effective communication with cancer patients and their family members when it comes to realistic care aims, and it must be noted that the given care is highly prominent in enhancing their quality of life (25).

Reluctance to participate in EOL discussions and issues in relation to death or dying is a common practice all over the world, especially in Asia (25). Although patients and family members claim that they want to be informed in all matters, they may be reluctant to initiate such discussions (8). Most cancer patients will not bring up the issues unless they are approached by healthcare providers with sensitive manners.

In the present study setting, it was presumed that some of the healthcare providers might be reluctant to initiate discussion regarding EOL issues, especially when the healthcare providers had different religious backgrounds. The results of the current study were in line with previous findings $(3,6)$. One of the most difficult areas of communication is to talk to the patients or their families from different sociodemographic cultures (6). Patients, their family members, and clinicians are often stressed out by the factors related to socio-demographic and cultures. These factors will influence the choice of treatment methods adopted for diseases (26).

The results of a study reported that most Chinese in Malaysia believed in the concept of yin-balance, which includes health (27). Meanwhile, the Malays follow health practices inherited from their ancestors, and the Indians are still practicing Ayurveda (26). To avoid cultural blind spots, doctors are required to comprehend the various cultural and traditional customs of their patients (28). A variety of cultural health beliefs, traditions, and practices occur among 
different ethnic groups in Malaysia (28). Patients might expect doctors to provide more detailed information on the diagnosis, treatment, and prognosis of the disease. Therefore, doctors must have the ability to deliver all kinds of information, including bad news.

\section{Limitations and Recommendations}

Generalization of the results of the present study to patients beyond the respondents assessed in this study should be made with caution because the respondents were collected from only one hospital. The convenience sampling method might have caused bias and limited generalization. The ability to make causal inferences was limited since the data were collected in a self-reporting manner and through cross-sectional analysis.

Although to the best of our knowledge, limited studies are examining the QOC between cancer patients and doctors regarding EOL care, there have been similar studies that have investigated this type of communication among a general patient population or patients with cancer. Future studies need to be performed to assess the measurement characteristics of this questionnaire and the responsiveness of this questionnaire to the interventions intended to improve the QOC.

It is highly recommended to incorporate cultural competency in training communication skills that focus on cancer patient care in continuous professional development for healthcare providers across every level of care, from primary to quaternary. Ultimately, the goal is to improve the quality of life of cancer patients.

\section{Conclusion}

The findings of this study indicated that age, income, education level, and employment were significantly related to the QOC between the patient and doctor. Consequently, it is essential for physicians and healthcare providers to understand the characteristics of the patients, consider the differences in each patient, and apply more effective communication. The provision of training in effective communication between patients and doctors or healthcare providers is crucial to improve communication, which will further improve the quality of care and quality of life among cancer patients.

\section{Acknowledgments}

The authors would like to express their gratitude to all cancer patients who participated in this study for their support.

\section{Footnotes}

Authors' contributions: M. ZH. designed and performed the paper, analyzed data, wrote the paper, and prepared the manuscript. MC. C. supervised the research and prepared the manuscript. LP. W. supervised the data analysis and prepared the manuscript. AM. AR. prepared the manuscript. LY. AA. prepared the manuscript preparation. S. M. prepared the manuscript.

Conflicts of interest: The authors declare that there is no conflict of interest.

Funding/Support: None.

\section{References}

1. Hulvat MC. Cancer incidence and trends. Surg Clin. 2020;100(3):469-81. doi: 10.1016/j.suc.2020.01.002

2. Schliemann, D., Ismail, R., Donnelly, M. Cancer symptom and risk factor awareness in Malaysia: findings from a nationwide crosssectional study. BMC Pub Heal. 2020;20:464. PMCID: PMC7137199 doi: 10.1186/s12889-020-08581-0

3. Distelhorst SR, Cleary JF, Ganz PA, Bese N, Camacho-Rodriguez $\mathrm{R}$, Cardoso F, et al. Optimisation of the continuum of supportive and palliative care for patients with breast cancer in lowincome and middle-income countries: executive summary of the Breast Health Global Initiative, 2014. Lanc Oncol. 2015;16(3):e137-e47. doi: 10.1016/S1470-2045(14)70457-7

4. Rajeshwari A, Revathi R, Narayanan Prasad NM. Assessment of distress among patients and primary caregivers: Findings from a chemotherapy outpatient unit. Ind J Palliat Care. 2020;26(1):42. PMCID: PMC7017693 doi: 10.4103/IJPC.IJPC_163_19

5. Walczak A, Butow PN, Bu S, Clayton JM. A systematic review of evidence for end-of-life communication interventions: who do they target, how are they structured and do they work? Patient Educa Couns. 2016;99(1):3-16. doi: 10.1016/j.pec.2015.08.017

6. Faller H, Weis J, Koch U, Brähler E, Härter M, Keller M, et al. Perceived need for psychosocial support depending on emotional distress and mental comorbidity in men and women with cancer. J Psychos Res. 2016;81:24-30. doi: 10.1016/j.jpsychores.2015.12.004

7. Betancourt JR, Green AR, Carrillo JE, Owusu Ananeh-Firempong I. Defining cultural competence: a practical framework for addressing racial/ethnic disparities in health and health care. Pub Heal Repor. 2016. PMCID: PMC1497553 doi: 10.1093/phr/118.4.293

8. Surbone A. Communication is the key: Can it be taught, learned and assessed? Ethics and Oncology. Ther, Care Res. 2018;19:2129. https://ixtheo.de/Record/1019117192

9. Brown O, Ham-Baloyi Wt, Rooyen Dv, Aldous C, Marais LC. Culturally competent patient-provider communication in the management of cancer: An integrative literature review. Glob Heal Act. 2016;9(1):33208. PMCID: PMC5134830 doi: 10.3402/gha.v9.33208

10. Sharif Nia H, Sharif SP, Esmaeili R, Goudarzian AH, Tahmasbi B, Yaghoobzadeh A, et al. Factors influencing the level of death depression in patients with cancer: a path analysis. J Mazand Uni Med Sci. 2017;26(145):318-31. http://jmums.mazums.ac.ir/article-1-9509-en.html

11. Epstein AS, Prigerson HG, O'Reilly EM, Maciejewski PK. Discussions of life expectancy and changes in illness understanding in patients with advanced cancer. J Clin Oncol. 2016;34(20):2398. PMCID: PMC4981977 doi: 10.1200/JCO.2015.63.6696

12. Mayer DK, Nasso SF, Earp JA. Defining cancer survivors, their needs, and perspectives on survivorship health care in the USA. Lancet Oncol. 2017;18(1):e11-e8. doi: 10.1016/S14702045(16)30573-3

13. Whitehead L, Walker DK. Communication skills training for health care professionals who work with cancer patients. AJN Amer J Nurs. 2019;119(7):45-52. PMCID: PMC6457800 doi: 10.1002/14651858.CD003751.pub3

14. Brueck M, Salib AM. Strategies for acing the fundamentals and 
mitigating legal and ethical consequences of poor physicianpatient communication. AMA J Eth. 2017;19(3):289-95. doi: 10.1001/journalofethics.2017.19.3.hlaw1-1703

15. Barracliffe L, Yang Y, Cameron J, Bedi C, Humphris G. Does emotional talk vary with fears of cancer recurrence trajectory? A content analysis of interactions between women with breast cancer and their therapeutic radiographers. J Psychos Res. 2018;106:41-8. doi: 10.1016/j.jpsychores.2018.01.004

16. Engelberg R, Downey L, Curtis JR. Psychometric characteristics of a quality of communication questionnaire assessing communication about end-of-life care. J Palliat Med. 2006;9(5):1086-98. doi: 10.1089/jpm.2006.9.1086

17. Witte RS, Witte JS. Statistics. John Wiley \& Sons: 11th Edition ed; 2017.

18. WHO. Process of translation and adaptation of instruments. http://wwwwhoint/substance_abuse/research_tools/translati on/en/. 2009.

19. Fournier E, Jooste V, Woronoff A-S, Quipourt V, Bouvier A-M, Mercier M. Health-related quality of life is a prognostic factor for survival in older patients after colorectal cancer diagnosis: A population-based study. Digest Liv Dis. 2016;48(1):87-93. doi: 10.1016/j.dld.2015.09.006

20. Tkatch R, Musich S, MacLeod S, Alsgaard K, Hawkins K, Yeh CS. Population health management for older adults: review of interventions for promoting successful aging across the health continuum. Geront Geriat Med. 2016;2:2333721416667877. PMCID: PMC5486489 doi: 10.1177/2333721416667877

21. Davis S. Communication Barriers Effects on Trust Between the Patient and Provider amongst Hispanic Communities, 2016. PMID: 26896341. doi: 10.1177/0009922816629760

22. Street Jr RL, Mazor KM. Clinician-patient communication measures: drilling down into assumptions, approaches, and analyses. Patient Educ Couns. 2017;100(8):1612-8. doi: 10.1016/j.pec.2017.03.021

23. Malhotra C, Farooqui MA, Kanesvaran R, Bilger M, Finkelstein E. Comparison of preferences for end-of-life care among patients with advanced cancer and their caregivers: a discrete choice experiment. Palliat Med. 2015;29(9):842-50. doi: 10.1177/0269216315578803

24. Hofmeister M, Memedovich A, Dowsett LE, Sevick L, McCarron T, Spackman E, et al. Palliative care in the home: a scoping review of study quality, primary outcomes, and thematic component analysis. BMC Palliat Care. 2018;17(1):1-7. PMCID: PMC5842572 doi: 10.1186/s12904-018-0299-z

25. Gao X, Sun F, Ko E, Kwak J, Shen H-W. Knowledge of advance directive and perceptions of end-of-life care in ChineseAmerican elders: The role of acculturation. Palliat Support Care. 2015;13(6):1677. doi: 10.1017/S147895151500067X

26. Broom A, Chittem M, Bowden V, Muppavaram N, Rajappa S. Illness experiences, collective decisions, and the therapeutic encounter in Indian oncology. Qualit Heal Res. 2017;27(7):95163. doi: $10.1177 / 1049732316648125$

27. Zhong R, Rau P-LP, Li S, editors. Measuring Chinese Medical Beliefs of Chinese Adults. International Conference on Cross-Cultural Design. Springer; 2016. http://dx.doi.org/10.1007/978-3-31940093-8_72

28. Abdullah N, Borhanuddin B, Patah AEA, Abdullah MS, Dauni A, Kamaruddin MA, et al. Utilization of complementary and alternative medicine in multiethnic population: The Malaysian Cohort Study. J Evid Based Integ Med. 2018;23:2515690X18765945. PMCID: PMC5922489 doi: $10.1177 / 2515690 \times 18765945$ 